\title{
PMU-based informational support of power system control tasks
}

\author{
P. I. Bartolomey, S. A. Eroshenko, S. I. Semenenko \\ \& A. A. Suvorov \\ Ural Federal University, Ekaterinburg, Russia
}

\begin{abstract}
Up-to-date wide area measurement systems (WAMS) based on phasor measurement units (PMU) appeared at the very end of the $20^{\text {th }}$ century. Under present-day conditions, WAMS serve as the basis for information-measuring systems, which significantly improve power system control and operation. In practice, WAMS are mostly used for power system stability control and transient monitoring and visualization. This paper discusses the new opportunities for power system control quality improvement, resulting from PMU application for power system steady-state parameters' assessment. Firstly, better control is provided by online equivalent circuit parameters' identification using PMU measurement data and taking into account FACTS and other shunt and series compensation equipment. Secondly, the paper addresses the problems of "nodal" identification, which have taken on great importance recently due to the intensive development of small-scaled distributed generation. Based on PMU measurements of nodal voltages and incident transmission lines' electric currents, one can obtain online steady-state load characteristics, which can be used for dispatch control applications. Moreover, PMUs provide superaccelerated power flow calculations, which are of crucial importance for emergency automation, adjusted for prior operation. Such principles of emergency automation consist of the quick determination of control actions, aimed at power system stability maintenance in cases of any programmed faults' occurrence. It is known that such control is carried out by means of power flow calculations based on remote metering data. The proposed application and allocation of PMUs in the power system by means of combinatorial matrix transformation to triangle form give the possibility to perform accelerated node-
\end{abstract}


voltage analysis without equivalent circuit simplification. All the calculations are verified using IEEE test networks.

Keywords: FACTS, long-distance transmission line, parameter identification, telemetry, PMU placement, accelerated power flow calculations.

\section{Introduction}

The application of up-to-date distributed information-measuring systems, based on phasor measurements, implemented in wide area measurement systems (WAMS) [1-6] as well as in developing WACS and WAPS systems, using similar technology, or their combinations, improve the level and the quality of power system control informational support. However, at the present time, WAMS' functional capabilities are underused.

This paper discusses the new possibilities of power flow control quality improvement using phasor measurements of power system operation parameters, collected from phasor measurement units (PMU).

Firstly, this refers to online equivalent circuit parameters identification, taking into account FACTS and other shunt and series compensation equipment of power transmission lines [7-10].

Comprehensive application of the data, collected from PMU, gives the possibility not only to determine reliable equivalent circuit parameters, but to handle various power system control tasks, including power system observability determination, measurement data authentication, at a new qualitative level. This also results in significant power flow calculation time reduction, which is of crucial importance for dispatch and emergency control on-line calculations.

It is known, that control quality is mostly determined by power system observability and power flow monitoring accuracy as well as by the accuracy of power transmission lines' physical parameters identification, such as impedance and admittance of power network elements, which strongly depend on each other as well as on loading and weather conditions.

Secondly, the paper illustrates that PMUs are able to provide power system observability and state estimation time reduction as well as accelerated calculations of power flow, which is highly important for emergency automation, using decision tables for preventive and emergency control. High cost of PMU devices led to the necessity to optimize PMU allocation in the power system. Earlier, in the scientific literature [3, 11-14], different PMU siting approaches were investigated. Most of them are aimed at optimization task solution, minimizing the amount of PMU devices and the corresponding expenses.

Further in the paper PMU allocation approach, based on the algorithm [15], is considered. Corresponding calculations provide power system observability as well as super-accelerated solution for nodal voltage equation set without power network dimensions reduction by means of admittance matrix triangulation, which is of great importance for power system emergency automation, using decision tables for emergency control. In such systems, control action amount should be identified within a short polling cycle time period, using current 
telemetry data, to ensure power system stability in case of any predefined emergency occurrence.

The results are compared with other PMU allocation algorithms. Corresponding case studies, using IEEE test networks, are provided [16].

\section{Transmission network parameters identification using PMU data}

Given the accurate information about electric current and voltage vectors, one can obtain adequate current-state power system equivalent circuit, which can be used for power system control purposes. In particular, if we know the complex values of currents and voltages at the transmission line terminals, we can obtain its equivalent circuit parameters - series resistance $R$ and reactance $X$, shunt conductance $G$ and capacitive susceptance $B$.

Such application of PMUs is extremely actual, because the errors in power system equivalent circuit parameters can be rather considerable, if they are taken by reference data. According to scientific researches, conducted earlier in USSR, reflecting the range of possible daily and seasonal conditions, power transmission line parameters can vary within relatively wide ranges (Table 1). More accurate transmission line parameters are followed by the accuracy improvement of any calculations, where these parameters are used. It is notable, that due to seasonal and daily conditions variation, equivalent circuit update should be carried out several times per day.

Table 1: Equivalent circuit parameters errors.

\begin{tabular}{|c|c|c|c|}
\hline $\begin{array}{c}\text { Equivalent } \\
\text { circuit } \\
\text { parameter }\end{array}$ & Error cause & Error value & $\begin{array}{c}\text { Power flow } \\
\text { influence on } \\
\text { error }\end{array}$ \\
\hline$R$ & $\begin{array}{c}\text { Skin effect and weather conditions neglecting } \\
R=R_{\left(20^{\circ}\right)}\left[1+0,004 \cdot\left(T^{\circ}-20\right)\right] .\end{array}$ & $-24 \ldots+8 \%$ & Strong \\
\hline$X$ & $\begin{array}{c}\text { Inaccuracy in transmission lines geometry } \\
\text { modeling. Multiple grounded overhead } \\
\text { protection cables, parallel circuits. }\end{array}$ & $-10 \ldots+8 \%$ & Weak \\
\hline$B$ & $\begin{array}{c}\text { Transmission line sag, overhead protection } \\
\text { cable grounding, parallel circuits, ground and } \\
\text { humid air conductivity neglecting. }\end{array}$ & $25 \ldots 30 \%$ & Strong \\
\hline$G$ & $\begin{array}{c}\text { Construction peculiarities, power flow and } \\
\text { atmospheric conditions }\end{array}$ & $1,5-3$ times & Very strong \\
\hline
\end{tabular}

An approach of equivalent circuit parameters identification, using current steady-state power flow parameters, is further considered. It is notable, that this approach provides reliable results whether there is series or shunt FACTS equipment in the power network or not.

A particular case is an overhead power transmission line. In case of 200 $300 \mathrm{~km}$ length, it can be approximated by symmetrical $\pi$-circuit model with an admissible error: 


$$
\begin{gathered}
Z_{12}=R_{12}+j X_{12}=\left(r_{\mathrm{o}}+j x_{\mathrm{o}}\right) L_{12}, Y_{12}=Z_{12}^{-1}=G_{12}-j B_{12} ; \\
Y_{10}=Y_{20}=G_{10}+j B_{10}=\left(g_{\mathrm{o}}+j b_{\mathrm{o}}\right) L_{12} / 2,
\end{gathered}
$$

where $r_{\mathrm{o}}, x_{\mathrm{o}}, g_{\mathrm{o}}, b_{\mathrm{o}}$ - specific line parameters per $1 \mathrm{~km}$ length; indexes 1 and 2 indicate sending and receiving ends of the line, respectively.

A transmission line can be also represented by four-terminal element. The relation between four-terminal parameters and power flow parameters is wellknown and can be presented by the following expressions:

$$
\left.\begin{array}{l}
\dot{U}_{1}=A_{11} \dot{U}_{2}+A_{12} \dot{I}_{2} \\
\dot{I}_{1}=A_{21} \dot{U}_{2}+A_{22} \dot{I}_{2}
\end{array}\right\},
$$

At the same time, conversion equations from matrix $\mathrm{A}$ to $(Z, Y)$ parameters and vice versa are the following:

$$
\left.\left.\begin{array}{l}
A_{11}=1+Z_{12} Y_{20} \\
A_{12}=Z_{12} \\
A_{21}=Y_{10}+Y_{20}+Z_{12} Y_{10} Y_{20} \\
A_{22}=1+Z_{12} Y_{10}
\end{array}\right\} \text { и } \begin{array}{l}
Y_{10}=\left(A_{22}-1\right) / A_{12} \\
Y_{20}=\left(A_{11}-1\right) / A_{12}
\end{array}\right\} .
$$

Taking into account, that in case of symmetrical four-terminal element $A_{11}=A_{22}$, and in the case of $\pi$-model $Y_{10}=Y_{20}=Y_{0}$, we obtain actual values of power transmission parameters using PMU measurement data, bypassing the definition of matrix A :

$$
Y_{0}=\frac{\dot{I}_{1}-\dot{I}_{2}}{\dot{U}_{1}+\dot{U}_{2}} \text { и } Z_{12}=\frac{\dot{U}_{1}-\dot{U}_{2}}{\dot{I}_{1}-Y \dot{U}_{1}},
$$

which can be further used for other power system control tasks, requiring equivalent circuit parameter calculations.

For instance, let's consider $200 \mathrm{~km} 500 \mathrm{kV}$ transmission line with the following initially given parameters: $Z=(5+j 72)$ Ohm, $Y=\left(2,24 \cdot 10^{-6}+j 3,60 \cdot 10^{-4}\right) \mathrm{S}, \quad \dot{U}_{1}=500 \angle 0^{\circ} \mathrm{kV}, \dot{I}_{1}=1000,12 \angle 0,0054^{\circ} \mathrm{A}$, $P_{1}=500 \mathrm{MW}, Q_{1}=-0,3$ MVAr.

The calculation results for the receiving end are:

$\dot{U}_{2}=487,26 \angle-8,39^{\circ} \mathrm{kV}, \dot{I}_{2}=1034,54 \angle-19,92^{\circ} \mathrm{A}, P_{2}=494,2 \quad \mathrm{MW}$ and $Q_{2}=100,9$ MVAr. 
Updated equivalent circuit parameters, taken as reference values for further calculations, were obtained during the inverse problem solution in accordance with expressions (4):

$$
Z_{\text {ref }}=(5.028+j 71.978) \mathrm{Ohm}, Y_{\text {ref }}=\left(2.05 \cdot 10^{-6}+j 3.59 \cdot 10^{-4}\right) \mathrm{S} .
$$

The results of PMU measurements variations within the ranges of $0.1 \%$ for electric current, $0.1 \%$ for voltages, $0.1 \%$ for phase angle show, that the relation between transmission line parameter errors and phase angle measurement error, obtained after equivalent circuit parameter identification, is linear. The most sensitive to phase angle measurement error parameter is active conductance, the next one - resistance. However, these parameters do not influence the result of steady-state power flow calculations greatly.

It should be mentioned that the most significant influence factors for phase angle measurements are the phase angle errors of instrument transformers, in particular - current transformer errors. The similar effect is given by inaccuracy of PMU synchronization. For example, if phase angle error equals to $\varepsilon \delta_{12}=0.2^{\circ}$, the corresponding equivalent circuit parameter identification error percentage is the following: $\varepsilon G=17 \%, \varepsilon R=4 \%, \varepsilon X=2 \%, \varepsilon B=1 \%$.

In general, proposed methodology provides equivalent circuit parameters identification with required accuracy.

\section{Accelerated steady-state power flow calculation using the PMU information}

Accelerating effect can be easily shown on the IEEE 14 bus power grid example, which is presented in Figure 1.

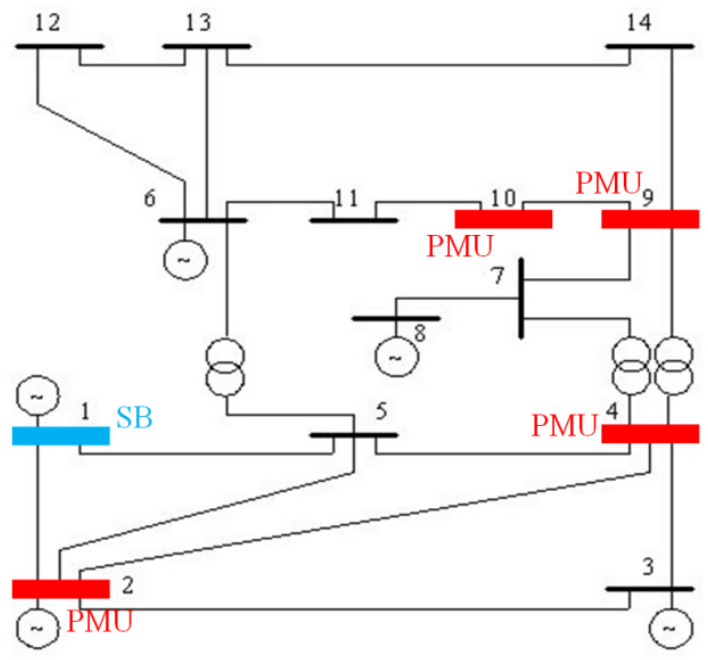

Figure 1: IEEE-14 power grid. Buses 1, 2, 4, 9, 10 are equipped with PMU. 
Let us assume that the set $p$, which includes the numbers of buses, where voltage measurements are carried out with PMU, is determined according to some criterion. The set, containing other buses, is referred to as $q$. For the case, presented in Figure 1, $p=(1,2,4,9,10), q=(3,5,6,7,8,11,12,13,14)$.

The electric current phasor measurements of the branches, which are incident to PMU-equipped buses, are not used, because in case of their fault, the observability of the power system may be lost. The application of these measurements should be discussed separately.

The initial admittance matrix $\mathbf{Y}$ from nodal analysis equation set is presented in Figure 2a as a dot diagram.

In accordance with the proposed algorithm the $\mathbf{Y}$ matrix can be transformed into the form, where triangular sub-matrix is separated after corresponding rearrangement of rows and columns. The resulting matrix is shown in Figure 2b.

$\{1\}\{3\}[1]\{2\}[2][3][4][5]\{4\}\{5\}[7][9][8][6]$

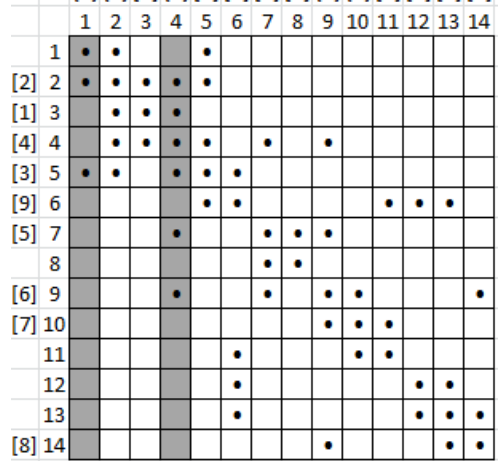

a

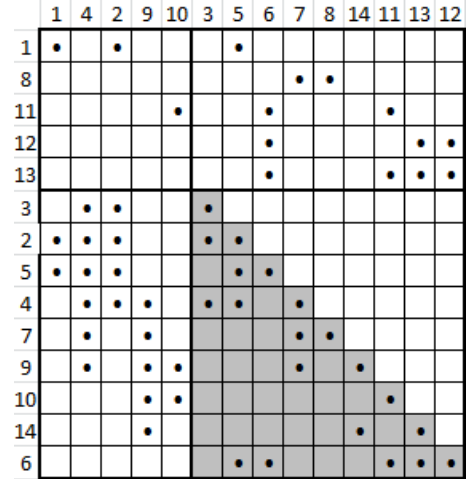

b

Figure 2: $\quad$ Y matrix dot diagrams.

So, if the nodal voltages of the set $p$ are measured by PMU devices, the voltages of the set $q$ set can be calculated by the sequential downward solution of complex equation with one unknown variable and subsequent substitution of that variable into the next equations. Power flow calculation time becomes incommensurably less comparing to conventional power flow equation system calculations. The $p$ set determination algorithm and sub-matrix formulation methodology are presented below.

Moreover, as it will be shown further, the proposed algorithm provides close to optimal number of buses with PMU, which were determined in [3, 11-13] for IEEE test networks [15]. To be more exact, the resulting amount of PMU devices is often less, because the information from PMU is used simultaneously with nodal injection data.

\subsection{PMU allocation for accelerated power flow calculations}

The placement and application of PMU, providing super-accelerated power flow solution without power network dimensions reduction, are proposed in this 
paper. Instead of conventional nonlinear system of $K$ complex equations ( $K$ - the number of buses), the $M \times M$ left triangular matrix is generated, where $M=K-N \quad(N-$ the number of PMU-equipped buses). The remaining $M$ equations are solved in a sequential order. Every equation has one unknown complex variable.

The PMU-equipped bus set was previously defined as $p, \operatorname{dim}(p)=N$. Consequently, the dimension of the set of buses without PMU is $\operatorname{dim}(q)=M$. Admittance matrix with self-admittances $Y_{i, i}$ (diagonal elements) and branch admittances $Y_{i, k}=g_{i, k}-j \cdot b_{i, k}$ can be divided into separate blocks as follows:

$$
\begin{array}{|l|l|}
\hline Y_{p p} & Y_{p q} \\
\hline Y_{q p} & Y_{q q} \\
\hline
\end{array} \times \begin{array}{|l|}
\hline U_{p} \\
\hline U_{q} \\
\hline I_{q} \\
\hline
\end{array}
$$

Nodal voltage equation system in currents is given below:

$$
\begin{aligned}
& Y_{p p} \cdot U_{p}+Y_{p q} \cdot U_{q}=I_{p}, \\
& Y_{q p} \cdot U_{p}+Y_{q q} \cdot U_{q}=I_{q} .
\end{aligned}
$$

Assuming that complex values $U_{p}$ are measured, the remaining bus voltages $U_{q}$ can be evaluated by solving the equation set (8), which can be given as follows:

$$
Y_{q q} \cdot U_{q}=I_{q}-Y_{q p} \cdot U_{p} .
$$

Vector $I_{q}$ components are calculated using active $P_{i}$ and reactive $Q_{i}$ power injections and voltages $V_{j}$ data, collected from the conventional telemetry (without phasor measurements). System (9) dimension is $\operatorname{dim}\left(Y_{q q}\right)=M \times M$.

If the rows and columns of matrix $\mathbf{Y}$ are rearranged according to the stated below algorithm, the $Y_{q q}$ matrix becomes triangular as shown in Figure 2. It should be noted again, that downward solution is carried out for complex equations with one unknown variable, which are further substituted into remaining equations. System

$$
Y_{q q}^{T} \cdot U_{q}^{T}=I_{q}^{T}-Y_{q p}^{T} \cdot U_{p}^{T},
$$

where T-index indicates triangular transformation result, has less computation time, than conventional one (9).

\subsection{Triangular sub-matrix generation algorithm}

1. If the slack bus with installed PMU was previously determined, the algorithm procedure begins with assigning the bus with number $\{1\}$. The corresponding column in matrix $\mathbf{Y}$ is removed. In our example, presented in Figure 2a, column 1 is highlighted. The same procedure should be performed with the buses, where PMUs are initially installed.

2. Minimal-rank node search. It is necessary to find the column with the maximum nonzero entries in the diagram (Figure 2a). In case of multiple maximal-rank buses, the leftmost one is chosen. That bus voltage is further considered as determinative and the corresponding matrix $\mathbf{Y}$ column is assumed to be evaluated. That column is then coloured (grey in our case) and numbered 
$\{2\}$, since number $\{1\}$ has been already assigned to the slack bus 1 . In the example the maximum rank bus is bus number 4 . According to the algorithm, the bus is assigned with number $\{2\}$, indicating that this bus voltage will be second determinative and the $4^{\text {th }}$ bus will be the $1^{\text {st }}$ in the new matrix (Figure $2 \mathrm{~b}$ ). The column is coloured, and all its elements are considered to be evaluated.

3. Equation with one unknown variable search. In the entire matrix, one should find the row with only one unknown voltage variable. Equation $i$ with one unknown variable $U_{j}$ gives the possibility to calculate the voltage, if the bus current injection $I_{j}$ is initially given. If an equation of that type exists, go to step 4. Otherwise, the equation with minimum unknown voltages should be determined. After that the leftmost maximum rank bus is defined (step 2).

In the example, the equation with one unknown variable is absent, so the equation with the minimum amount of unknown voltages should be found (row 3 if searching downwards from top). Obviously, in that row the maximum rank column is column 2. It is assigned with number $\{3\}$. That shows that this variable will be the $3^{\text {rd }}$ determinative voltage and the column will be the $3^{\text {rd }}$ in the new matrix (Figure 2b). The column is highlighted with colour and all its entries are assumed to be known.

4. The determined row is assigned with number [1] and the same number is assigned to the voltage (column), calculated from that row; the column is highlighted with color. Other rows and columns are assigned with numbers [2], [3], etc. in accordance with stated rules of the algorithm. The algorithm goes to step 2 until all the columns are passed. The further actions are carried out according to subsequent steps, described above.

The results for the 14 bus test network are presented in Figure 2b, where we can see the new rows and columns numeration that provides the triangular submatrix assignment.

5. Rows and columns numeration analysis. After the numeration and determinative voltages are obtained, the new diagram is divided into submatrixes. The upper rows of the obtained matrix are the initial matrix rows, that haven't obtained the indexation. Subsequent rows are the rows of initial matrix in the new order $\{1\},\{2\}$, etc. The columns are presented in the new order too: firstly, the determinative nodes $\{\mathrm{i}\}$, and then the remaining ones $\{\mathrm{j}\}$. As a result, we generate the new matrix with assigned triangular sub-matrix, which is presented in the Figure $2 b$.

\subsection{PMU devices number minimization}

PMU gives the possibility to obtain information not only about the bus voltage, but also about electric current values of transmission lines, that are incident to the bus. The application of such data results in PMU amount reduction in comparison with the previous case, where only the voltage data was used.

It can be easily illustrated for the same test network (Figure 1).

In accordance with the developed algorithm, the calculation steps are the following: 
1. Voltage values $\dot{U}_{2}^{(p)}, \dot{U}_{5}^{(p)}$ are calculated through the voltage drops in transmission lines 1-5 and 1-2 using PMU1 measurements: $\dot{U}_{1}^{P M U}, \dot{I}_{1-5}^{P M U}, \dot{I}_{1-2}^{P M U}$. 2. Voltage values $\dot{U}_{3}^{(p)}, \dot{U}_{7}^{(p)}$ and $\dot{U}_{9}^{(p)}$ are calculated through the voltage drops in transmission lines 4-3, 4-7 and 4-9 using PMU4 measurements: $\dot{U}_{4}^{P M U}, \dot{I}_{4-3}^{P M U}$, $\dot{I}_{4-7}^{P M U}, \dot{I}_{4-9}^{P M U}$.

3. Voltage values $\dot{U}_{11}^{(p)}, \dot{U}_{12}^{(p)}$ and $\dot{U}_{13}^{(p)}$ are calculated through the voltage drops in transmission lines 6-11, 6-12 and 6-13 using PMU6 measurements: $\dot{U}_{6}^{P M U}$, $\dot{I}_{6-11}^{P M U}, \dot{I}_{6-12}^{P M U}, \dot{I}_{6-13}^{P M U}$.

4. $\dot{U}_{8}^{(p)}$ is calculated using initially known load current value $\dot{I}_{8}$ and calculated voltage $\dot{U}_{7}^{(p)}$.

5. $\dot{U}_{10}^{(p)}$ is calculated from the complex equation using initially known load current value $\dot{I}_{10}$ and the calculated voltages $\dot{U}_{9}^{(p)}$ and $\dot{U}_{11}^{(p)}$.

6. $\dot{U}_{14}^{(p)}$ is also calculated by solving one complex equation using initially known load current value $\dot{I}_{14}$ and the calculated voltage values $\dot{U}_{9}^{(p)}$ and $\dot{U}_{13}^{(p)}$.

Thus, power flow calculation, that are carried out without solving massive nonlinear equation system $(26 \times 26)$, for the given test network require only three PMUs installation in buses 1, 4 and 6 .

It is important to say, that proposed algorithm provides not only power system observability and accelerated power flow calculations, but also PMU devices amount minimization.

The algorithm (A1) operation will be further demonstrated on IEEE-30 international test network, containing 30 buses. Figure 3 presents the resulting admittance matrix dot diagram after row and column rearrangement. As we can see from Figure 3 , the $p$ set is $p=(1,2,4,6,10,15,18,22,28,29)$ and its $\operatorname{dimension}$ is $\operatorname{dim}(p)=10$.

An interesting result was obtained for IEEE-57 test network [15]. The set $p=(1,3,4,6,7,9,10,11,12,14)$ includes 10 measurements $(\operatorname{dim}(p)=10)$, which is less than resulting amount of PMUs from other existing PMU placement algorithms A4 [11], A5 [3], A6 [13], A7 [12]. The comparison is presented in Table 1.

Since PMU provides not only bus voltage magnitude and its phase, but also complex values of bus, linear and transformer current injections, the necessity of accelerated power flow calculation algorithm improvement arises.

Developed algorithm A2 is noted for the application of PMU electric current measurements along with conventional bus current injections remote meters (RM). One more modification A3 is based only on PMU current and voltage measurements. Figure 4 demonstrates RM and PMU allocation for 14-IEEE test network, which results from A2 algorithm operation. As we can see, only 2 PMU devices are required in buses 4 and 6 along with 4 RM meters in buses 1, 8, 10 and 14. 


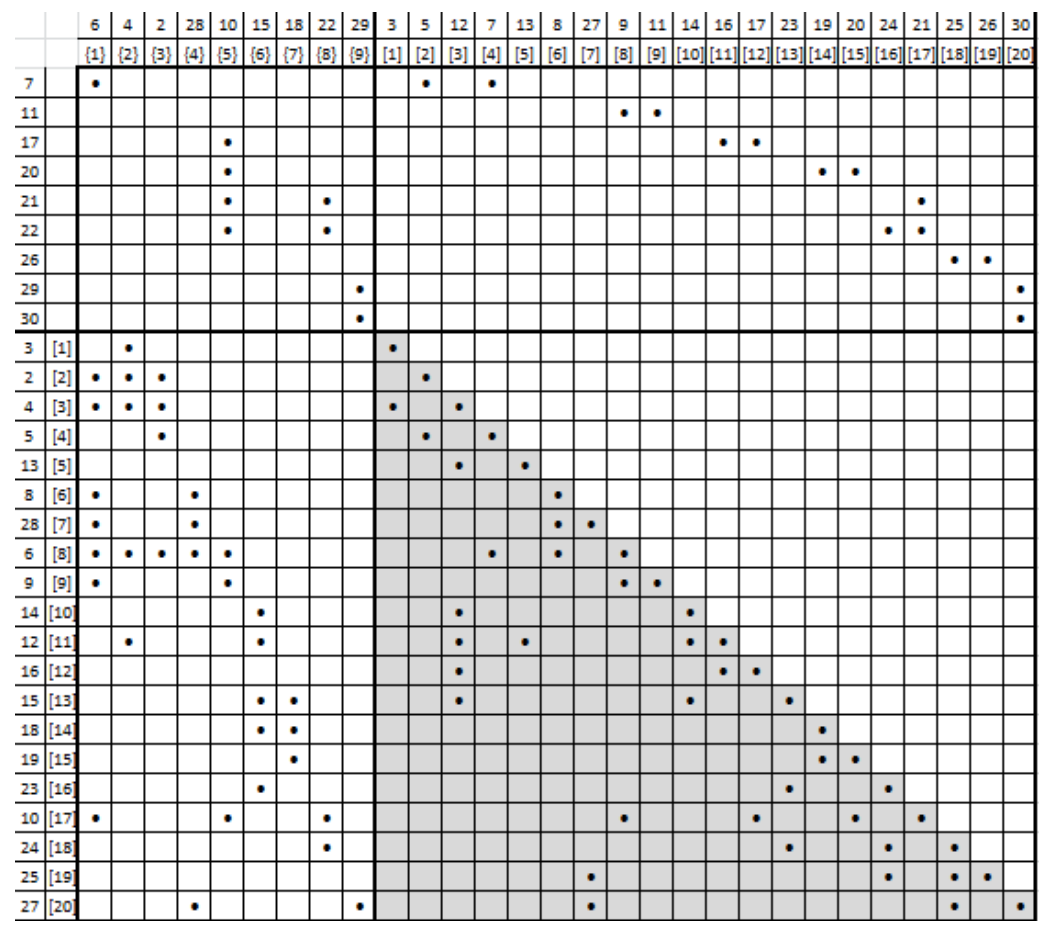

Figure 3: $\quad$ Resulting Y dot diagram for IEEE-30 test network.

Special attention must be given to proposed algorithm comparison with other existing techniques [3,11-13]. The most demonstrative is comparison between A1 and A4 [11] algorithms. The latter one also results in accelerated power flow calculations. It can be seen, however, that in A4 PMU costs, essential to ensure power system observability, are higher, because conventional meters are discarded. Comparative evaluations are presented in the following table.

Table 2: Comparison results.

\begin{tabular}{|c|c|c|c|c|c|c|c|c|}
\hline \multirow{2}{*}{ Power grid } & \multicolumn{9}{|c|}{ Algorithms } \\
\cline { 2 - 9 } & A1 [15] & A2 & A3 & A4 [11] & A5 [3] & A6 [13] & A7 [12] & A8 [4] \\
\hline IEEE-14 & $\mathbf{4}$ & 2 & 4 & $\mathbf{4}$ & 4 & 4 & 4 & - \\
\hline IEEE-30 & $\mathbf{9}$ & - & - & - & 10 & 10 & - & - \\
\hline New England & $\mathbf{9}$ & 8 & 8 & $\mathbf{8}$ & - & - & - & - \\
\hline IEEE-57 & $\mathbf{1 0}$ & 9 & 15 & - & 17 & 17 & 17 & 13 \\
\hline IEEE-118 & $\mathbf{2 8}$ & - & - & - & 32 & 32 & 32 & - \\
\hline
\end{tabular}




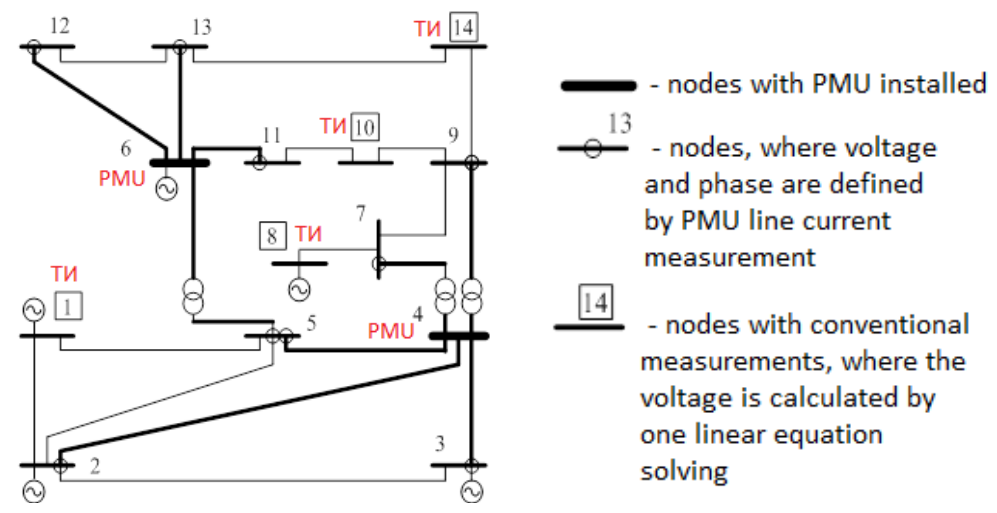

Figure 4: $\quad$ PMU and RM allocation according to A2 algorithm.

The proposed algorithm is based on the topological information only and doesn't consider the nodes with zero injections.

\section{Conclusions}

1. In spite of acceptable testing results, nowadays, the implementation of power network parameter identification function is limited due to the lack of PMU devices in the power system. The solution may be achieved if all available up-todate metering equipment is used. Moreover, this equipment should be provided with time synchronization functions similarly to PMUs with the possibility of data transmission to the information processing centers.

2. PMU devices placement based on the sub-matrix $Y_{q q}^{T}$ triangulation provides both the power system observability and super accelerated power flow calculations. It is not required to find the solution for massive nonlinear equation system in the proposed algorithm; equations have only one complex unknown variable and are solved sequentially. Moreover, the resulting number of PMU devices turned out to be minimal.

\section{References}

[1] A. G. Phadke, "Synchronized Phasor Measurements. A Historical Overview," in Proc. 2002, IEEE/PES Transmission and Distribution Conference, vol. 1, pp. 476-479.

[2] A. G. Phadke, J. S. Thorp, Synchronized Phasor Measurements and Their Applications. Power Electronics and Power Systems, 2008, Springer Science+Business Media, LLC, 2008, p. 245.

[3] Y. J. Yoon. Phasor Measurement Units for Large Scale Power System State Estimation. A Thesis for the degree of Master of Science. Texas A\&M University, December 2005. 
[4] M. Korkali and A. Abur, "Optimal Deployment of Wide-Area synchronized measurements for fault-location observability", IEEE Transactions on Power Systems, February, 2013, Vol. 28, No. 1, pp. 482489.

[5] P. I. Bartolomey, E. N. Begalova, A. V. Pazderin, E. A. Plesnyaev, “A Three-Stage Improving of Power System Operating Modes Measuring Data Validation," in Proc. of International Conference on Advanced Power System Automation and Protection at April 24-27, 2007 (APAP 2007), Jeju, Rep. Korea, 2007, pp. 173-176.

[6] Chusovitin P., Pazderin A., Implementation of Power System Model Identification for Locating In-phase Generators. Proceedings of 3rd IEEE PES Innovative Smart Grid Technologies Europe (ISGT Europe). Berlin, 2012.

[7] Bartolomey P.I., Eroshenko S.A., Lebedev E.M., Suvorov A.A., New Information Technologies for State Estimation of Power Systems with FACTS, Proc. of the International conf. "3rd IEEE PES Innovative Smart Grid Technologies Europe (ISGT Europe)”, October 14-17, 2012, Berlin, Germany.

[8] Di Shi, Daniel J. Tylavsky, Kristian M. Koellner, Naim Logic and David E. Wheeler, "Transmission line parameter identification using PMU measurements," Euro. Trans. Electr. Power 2011:21, pp. 1574-1588.

[9] Xiaomeng Bian, X. Rong Li, Huimin Chen, Deqiang Gan and Jiaju Qiu, "Joint Estimation of State and Parameter With Synchrophasors - Part I. State Tracking; Part II. Parameter Tracking," IEEE Trans. on Power Systems, vol. 26, No. 3, pp. 1196-1208 and pp. 1209-1220, Aug. 2011.

[10] Jian Chen and Ali Abur, "Placement of PMUs to Enable Bad Data Detection in State Estimation," IEEE Trans. on Power Systems, vol. 21, No. 4, November, 2006, pp. 1608-1615.

[11] T. L. Baldwin, L. Mili, M. B. Boisen, R. Adapa, “ Power System Observability With Minimal Phasor Measurement Placement", IEEE Transactions on Power Systems, vol. 8, No. 2, May 1993, pp. 707-715.

[12] Devesh Dua, Sanjay Dambhare, Rajeev Kumar Gajbhiye and S. A. Soman, "Optimal Multistage Scheduling of PMU Placement: An ILP Approach", IEEE Transactions on Power Delivery, vol. 23, No. 4, October, 2008, pp. 1812-1820.

[13] Nikolaos M. Manousakis and George N. Korres, "A Weighted Least Squares Algorithm for Optimal PMU Placement." Transactions on Power Systems, vol. 28, No. 3, August 2013, pp. 3499-3500.

[14] B. Milosevic and. M. Begovic "Nondominated Sorting Genetic Algorithm for Optimal Phasor Measurement Placement", IEEE Transactions on Power Systems, vol. 18, No. 1, February, 2003, pp. 69-75.

[15] P. I. Bartolomey, "Placement telemeasurements for on-line calculation of steady state regimes", Proceedings of the Ural State Technical University, Ekaterinburg, No. 2(10), 2000, pp. 32-37.

[16] "Power System Test Archive", http://www.ee.washington.edu/research/ pstca/index.html 